\title{
GOLD PRICE FORECASTING USING BOX-JENKINS METHOD
}

\author{
NUR ATIKAH KHALID AND NURFADHLINA ABDUL HALIM*
}

\author{
School of Informatics and Applied Mathematics \\ Universiti Malaysia Terengganu, 21030 Kuala Nerus, Terengganu
}

*Corresponding author: lina@umt.edu.my

\begin{abstract}
In general, the nature of gold that acts as a hedge against inflation and its stable price over the course of the financial crisis has made it a unique commodity. Price forecasts are a must for gold producers, investors and central bank to know the current trends in gold prices. Forecasting the future value of a variable is often done with time series analysis method. This study was conducted to determine the best model for forecasting gold commodity prices as well as forecasting world gold commodity prices in 2018 using Box-Jenkins approach. The data used in this study was obtained from Investing.Com from 2015 until 2017. This study shows that ARIMA $(1,1,1)$ is the best model to predict gold commodity prices based on Mean Absolute Percentage Error (MAPE). MAPE value for ARIMA $(1,1,1)$ is $0.02 \%$, where this value proves that forecasting using $\operatorname{ARIMA}(1,1,1)$ is the best forecasting because MAPE value is less than $10 \%$.
\end{abstract}

Keywords: Box-Jenkins, ARIMA, Gold Price Forecasting, Stationary

\section{Introduction}

Gold is a unique commodity due to its ability to act as a hedge against inflation as well as an investment asset. Gold is the only commodity that retains value during the financial and economic crisis period. One of the reasons investors are interested in investing in gold is gold prices never fall. Besides, the purchase of gold by China and India is one of the factors that cause investors to be interested in investing in gold (Kusumadewi, 2014). On top of that, future inflation threats caused by the printing of monetary policy developed by central bank have led to the devastation of global currency exchange rate which has affected the rise or fall in gold prices. Furthermore, other factors affecting the rise or fall in gold prices are financial crisis, rising gold demand in the market, dollar exchange rate, oil prices and world political situation. Gold price forecasting is an important knowledge for every investor to know to determine the trend of future gold prices for investment purposes
(Kusumadewi, 2014).

Box-Jenkins method was used in this study to forecast the gold prices for investment purposes. Box-Jenkins approach is synonymous with Autoregressive Integrated Moving Average (ARIMA) modeling. Initially, this approach was introduced by George E. P. Box and Gwilym M. Jenkins in 1976. They provided a comprehensive explanation of the techniques of analyzing time series data to be used in the ARIMA univariate model. ARIMA modeling is usually used for time series analysis, predictions and controls. In the context of the Jenkins metric method, there are several basic models involved, which are Autoregressive (AR), Moving Average (MA) and Autoregressive Moving Average (ARMA). The combination between AR and MA produces ARMA, which is a model class used to analyze stationary univariate data series. When the stationarity assumption of a variable is not met, ARIMA modeling has been generated to overcome the case. 
Through this formulation, data series need to be differenced to achieve stationary conditions (Mohd Alias, 2011). A nonstationary data series needs to be differenced to the stationary state because the main conditions for using the Box-Jenkins method are that the data must be stationary (Ali et al.,
2016). For further understanding, BoxJenkins method can be referred to in Figure 1 (flow chart for Box-Jenkins method) below.

\section{Materials and Methods}

Algorithm and Flow Chart for BoxJenkins Method

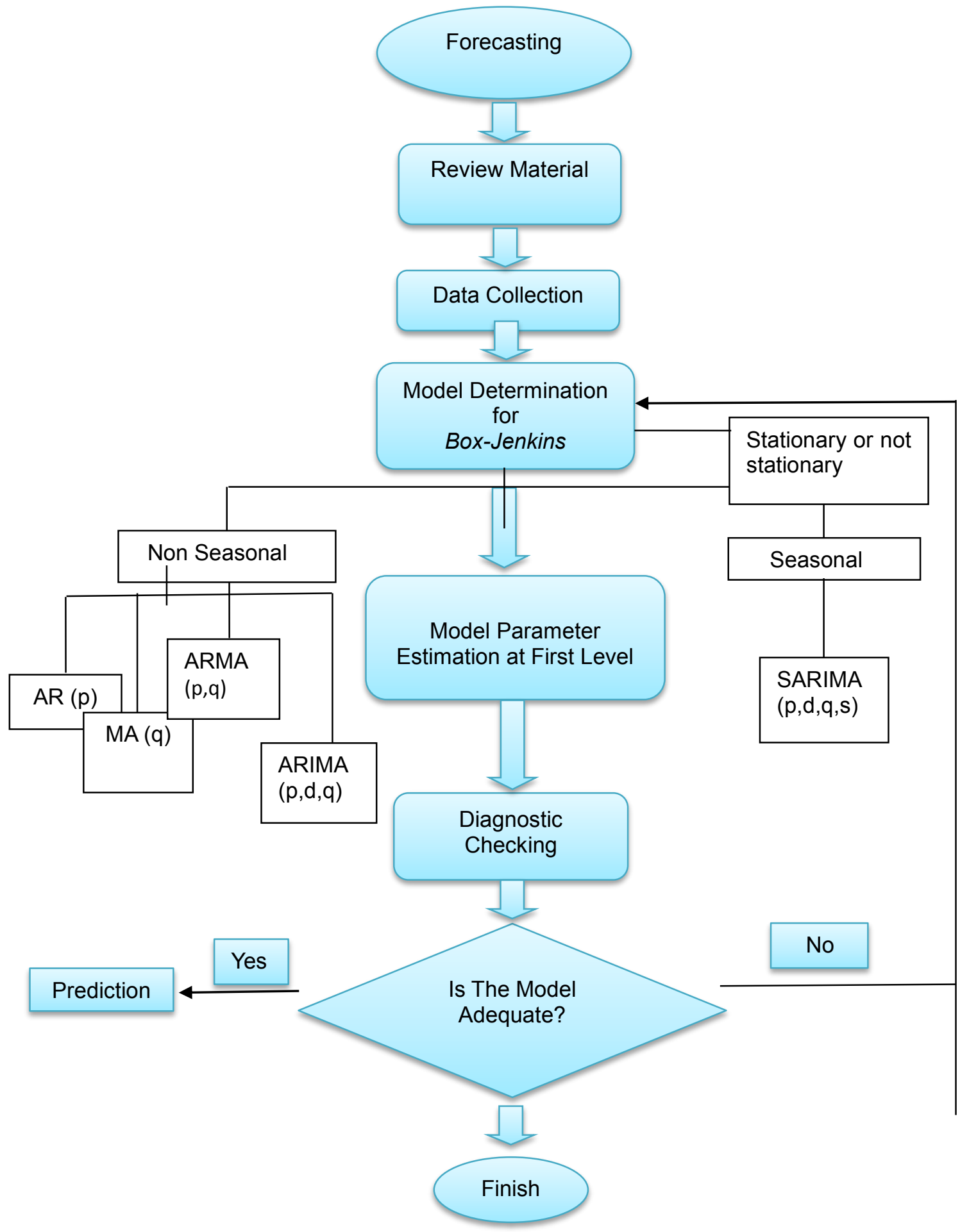

1.1 Flow Chart for Box-Jenkins Method 


\section{ARIMA Model}

This study used daily data from 1 January 2015 to 31 December 2017 to forecast world gold commodity prices for 2018. Data was obtained from Investing.Com website. This study used ARIMA model to forecast world gold commodity prices for 2018 .

This study used Autoregressive Integrated Moving Average (ARIMA) because the data was not stationary. BoxJenkins method was used to represent ARIMA $(p, d, q)$ where $d$ indicates the differentiation levels involved to reach stationary state used in this series. $p$ represents the number of Autoregressive
(AR) terms in a series and $q$ represents the number of Moving Average (MA) terms in a series. In this formulation, the time series data must go through differentiation process first to achieve the stationary state. ARIMA $(p, d, q)$ model can be written as follow:

$$
\phi_{p}(B)(1-B)^{d} y_{t}=\mu+\theta_{q}(B) \varepsilon_{t}
$$

where, $y_{t}$ is the original time series, $\phi_{p}$ is the autoregration parameter, $B$ is backward shift operator, $d$ is the degree of difference, $\mu$ is constant, $\theta_{q}$ is the moving average parameter and $\varepsilon_{t}$ is the error value at time $t$. Table 1 below shows recognition process for ARIMA model.

Table 1: Recognition Process for ARIMA Model

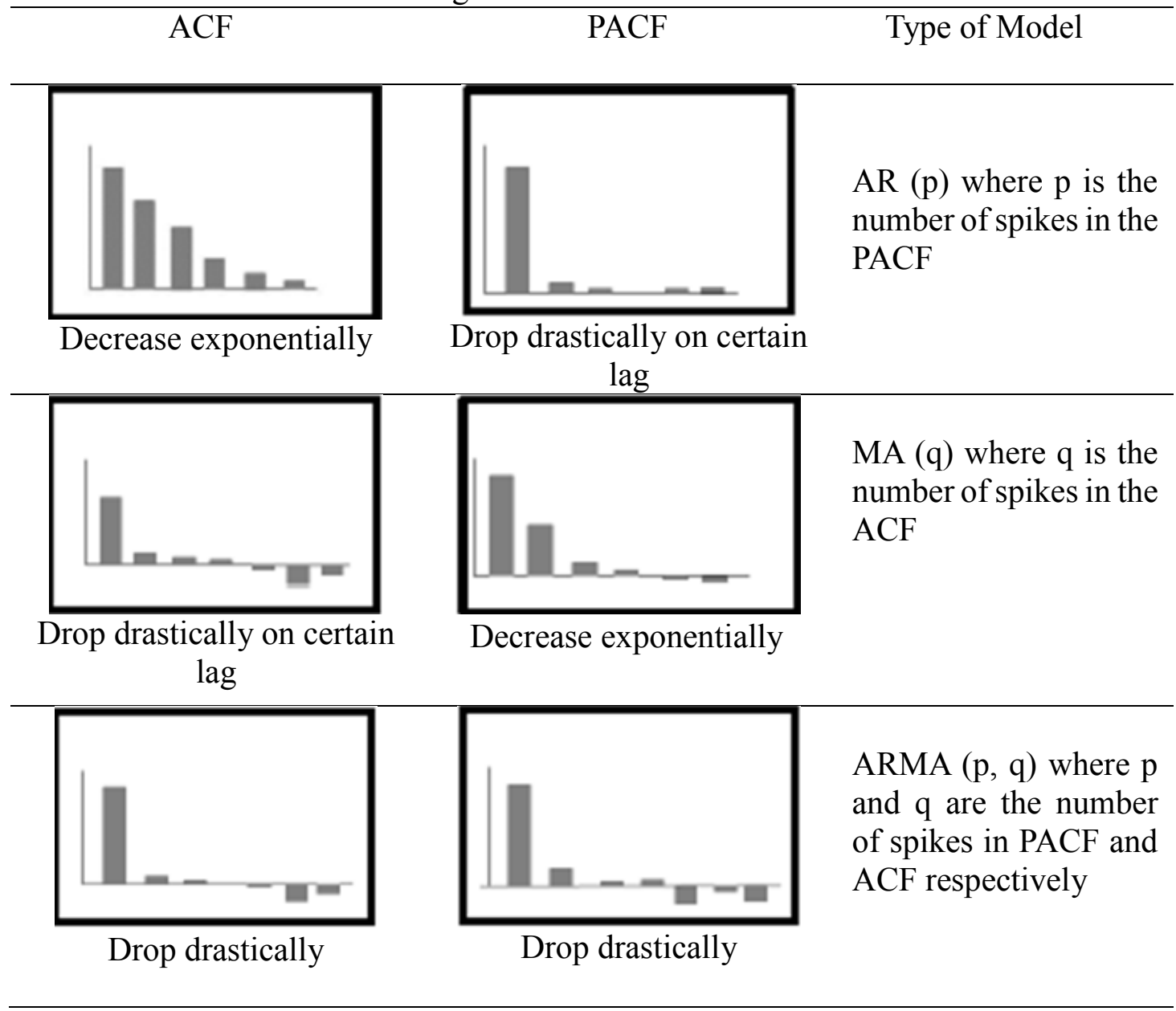

To facilitate readers to identify the values of $\mathrm{p}$ and $\mathrm{q}$, the following guidelines were used:

i) If the autocorrelation function $(\mathrm{ACF})$ decreases exponentially and the partial autocorrelation function (PACF) has spikes, the model used is AR. In this case, the model stage of AR (or $p$ value) is equal to the 
significant number of spikes in the PACF.

ii) If the partial autocorrelation function (PACF) decreases exponentially and the autocorrelation function (ACF) has spikes, the models used is MA. The model stage of MA (or $p$ value) is equal to the significant number of spikes in the ACF.

iii) If both autocorrelation function (ACF) and partial autocorrelation function (PACF) have

unorganized pattern, then the model used is ARMA model where the model stage (or $p$ value and $q$ value) is equal to the significant number of spikes in the PACF and ACF.

\section{Model Parameter Estimation at First Level}

The calculation that has been done to get the parameter value for any model is known as parameter estimation. There are two ways to estimate parameter, namely try and error method and fix iteratively. Through try and error method, testing towards few different values is done and selection of one value which can minimise total mean square error is chosen. Whereas through fix iteratively method, initial assessment selection is done and then the computer program refines iteratively the estimates.

\section{Diagnostic checking}

$$
Q=(T-d) \sum_{k=1}^{h} r_{k}^{2}
$$

which is scattered almost as a chi-square distribution with $(h-p-q)$ degrees of freedom where, $T$ is the number of observations in the time series, $h$ is maximum lag tested, $p$ is the highest stage of autoregration parameter in model, $q$ is the highest stage of moving average parameter in

$$
Q^{*}=T(T+2) \sum_{k=1}^{h} \frac{r_{k}^{2}}{T-k}
$$

and is also distributed as a chi-square distribution with $(h-p-q)$ degrees of freedom.
Diagnostic checks are conducted to prove that the model used is adequate. If the model fails in a diagnostic test, the model will be rejected. Results at this stage can show how a model can be improved. Q-Box Compression Value was used as a diagnostic check for this study.

Box-Jenkins framework assumes that the residuals (term errors) are unrelated to one another, and it is assumed that there is no systematic pattern in residuals. In other words, the error is considered to be normal, freely and randomly with zero mean and variance $\sigma_{\varepsilon}^{2}$ or can be shortly defined as $\varepsilon_{t} \sim\left(0, \sigma_{\varepsilon}^{2}\right)$. Therefore, if the error behavior does not meet these requirements, the model is said to be uncertain or of wrong specification. Incorrect specification is a symptom showing that important parameters may have been ignored or other unimportant parameters have been included in the model. The easy procedure for checking the wrong specifications is to check the presence of a correlation between the errors performed by calculating the chi-squared values in terms of errors. This test procedure is usually known as portmanteau test and this test is also a model validation. Model validation is also known as a test for wrong specifications among modelers. Statistical tests are given as: model, $r_{k}$ is k-th sample autocorrelation of error terms and $d$ is degrees of freedom of the differential used to the original data.

The latest version of trusted alternatives closer to the chi-square distribution of this set is the Ljung-Box statistics provided as:

Statistics show if each $r_{k}$ approaches zero, then $\mathrm{Q}$ or $Q^{*}$ each will becomes small and so big $r_{k}$ then $\mathrm{Q}$ or $Q^{*}$ will also be big. 
If the error is white noise, statistics $\mathrm{Q}$ or $Q^{*}$ are scattered as $X^{2}$ with $(h-p-q)$ degree of freedom. If the calculated value of $X^{2}$ is greater than the tabulated value of $X^{2}$ for $(h-p-q)$ degree of freedom, then reject $H_{0}$ (which shows the error is white noise) and accept $H_{1}$ (which shows the error is not white noise). By accepting $H_{1}$, the model is considered as wrong specification or inadequate to be considered. Similarly, if statistics $\mathrm{Q}$ is smaller than tabulated $X^{2}$ with $(h-p-q)$ degree of freedom, then accept $H_{0}$ (model is adequate).

If $H_{0}$ is rejected (the model is considered as wrong specification), then the best way is to try other model variations. In Box-Jenkins method, the possibility of two or more models having the same decision to accept $H_{0}$ is high. Therefore, in selecting the best model based on any condition, one needs to add decision criteria with other statistics such as Akaike Information Criterion (AIC), Bayesian Information Criterion (BIC) or Mean Square Error (MSE). Once again, when deciding on the best model, the parsimony concept is best suited because the simpler model is usually the preferred choice.

After these three stages have been done successfully, then forecasting can be done. In fact, this forecasting is a translation of equations based on the coefficients obtained so that the future determinations can be made. Forecasting can be determined whether it is good or not by calculating an absolute mean percentage error (MAPE). The error test is a measure of accuracy forecasting conducted. MAPE can be calculated as follows:

$$
M A P E=\sum_{t=1}^{n} \frac{\left|\left(\frac{e_{t}}{y_{t}}\right) * 100\right|}{n}
$$

where $n$ represents effective data point, $e_{t}$ represents the error which can be calculated as actual minus forecasted value and $\mid\left(\frac{e_{t}}{y_{t}}\right) *$ $100 \mid$ is defined as an absolute percentage error calculated on the predicted value for a particular forecasting method. The accuracy of the forecasting can be determined through the percentage of MAPE obtained through a forecasting as shown in Table2.

Table 2: MAPE Forecasting Power

\begin{tabular}{ll}
\hline MAPE & Forecasting Power \\
\hline$<10 \%$ & Very accurate forecasting \\
\hline $10 \% \sim 20 \%$ & Good forecasting \\
\hline $20 \% \sim 50 \%$ & Reasonable forecast \\
\hline$>50 \%$ & Weak and inaccurate forecasting \\
\hline
\end{tabular}

\section{Results and Discussion}

Based on time series plot of real value data in Figure 1, it was found that data did not show seasonal pattern. This is because observations did not repeat at regular intervals. Based on Figure 2, observation of the analysis of autocorrelation function (ACF) performed showed that the time series data was not in a stationary state. This can be seen clearly through slowly shrinking ACF plots. This shows that mean and variance for this time series data were not constant all the time $t$. Accordingly, differentiating processes need to be done to ensure that this series of time becomes stationary. Time series achieved stationary state after differentiation for the first time was done. 


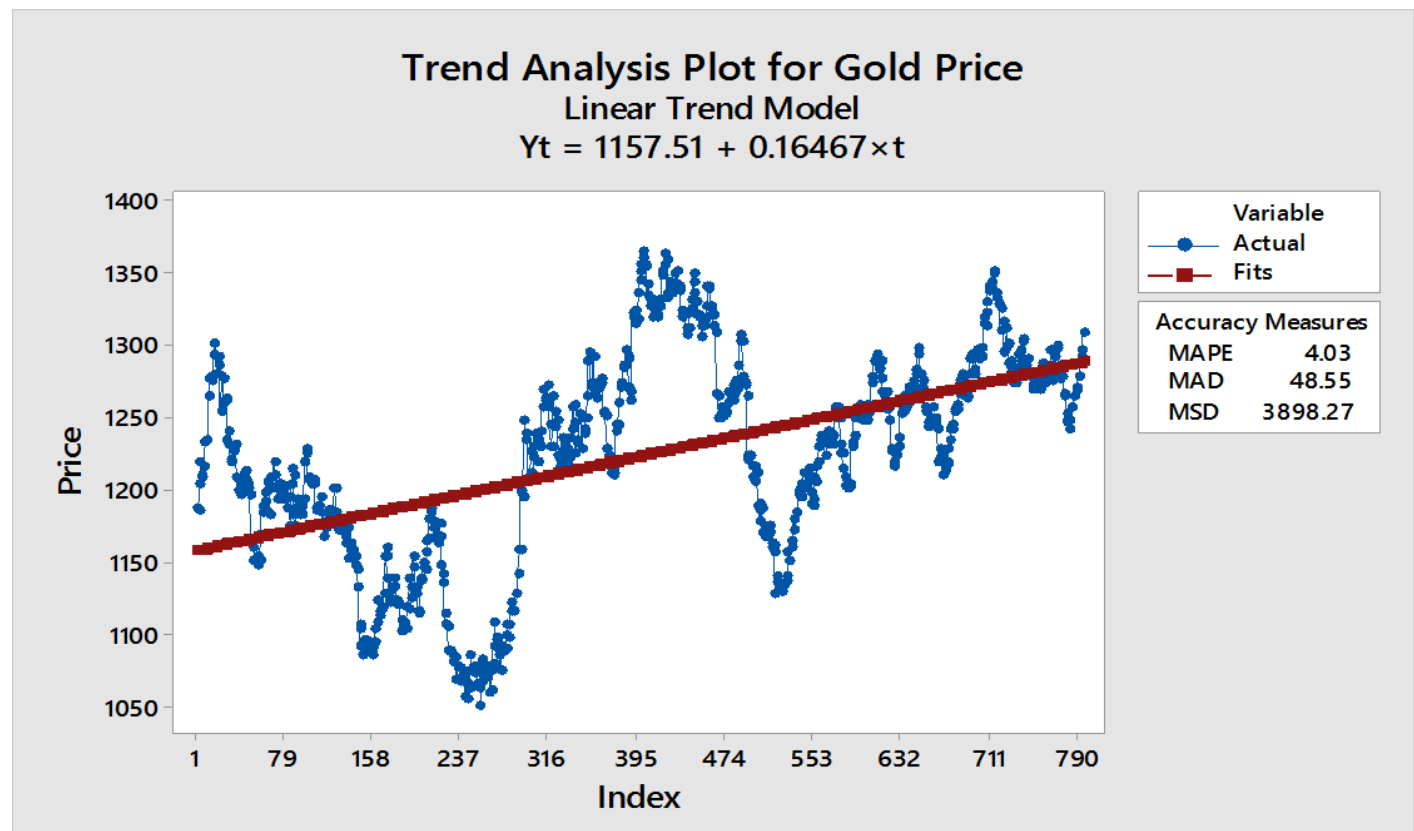

Figure 1: Trend Analysis Plot for Gold Price from January 2015 to December 2017

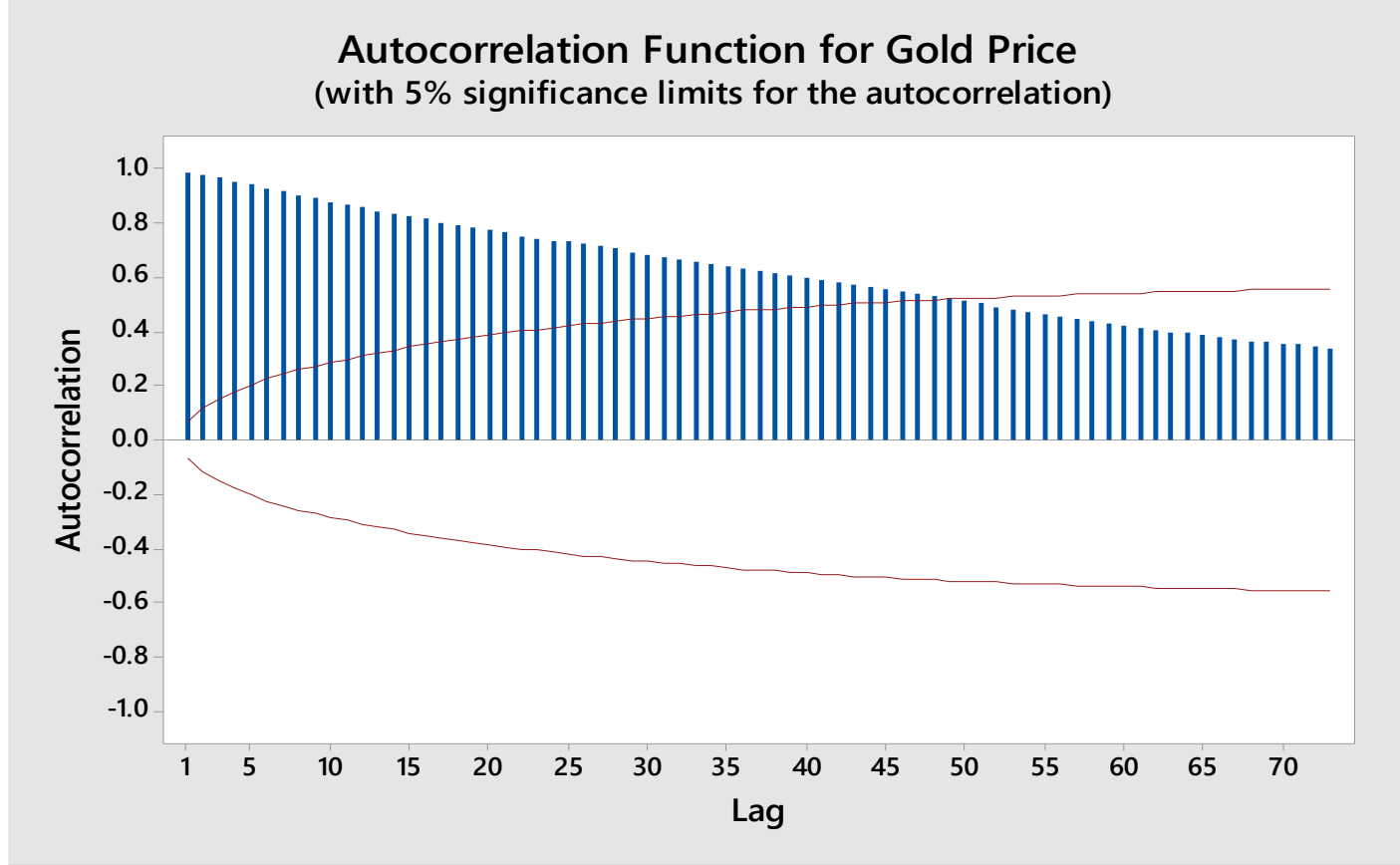

Figure 2: Autocorrelation Function (ACF) for Gold Price from January 2015 to December 2017 


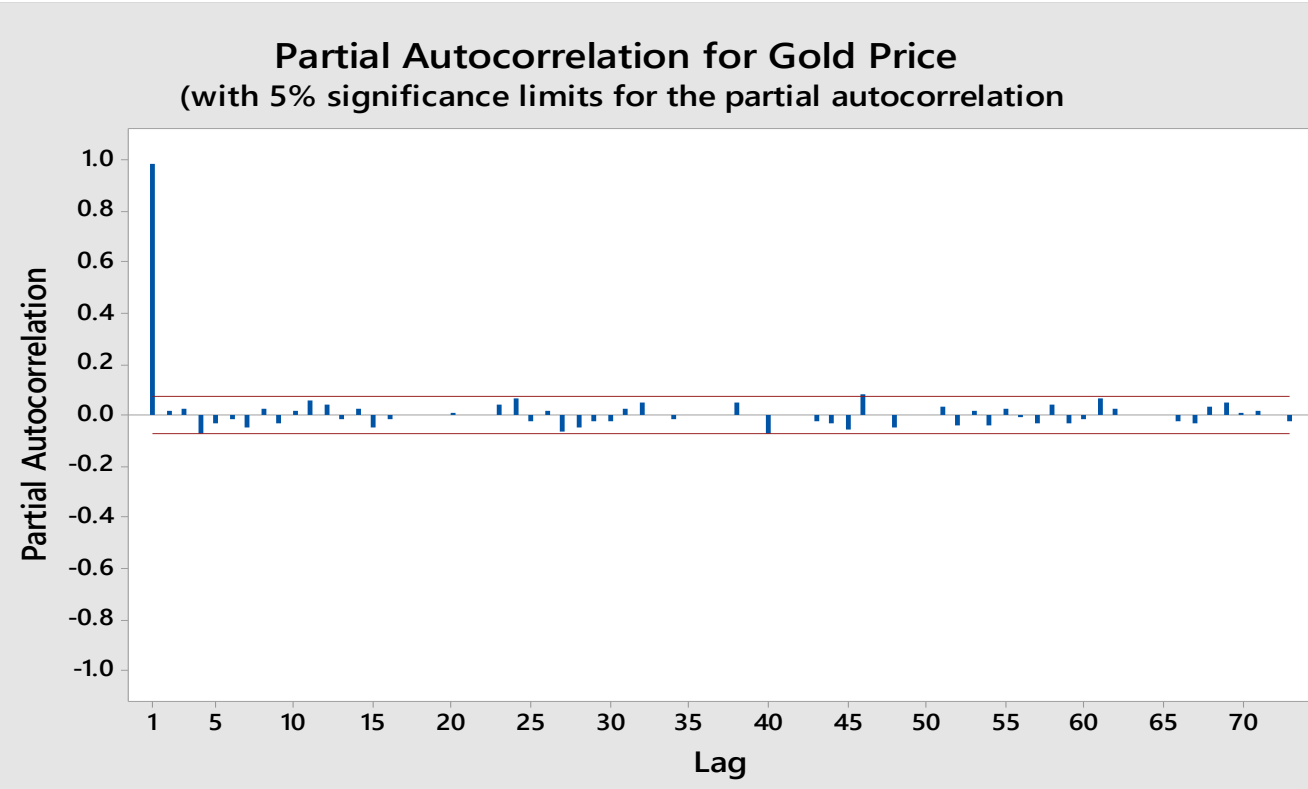

Figure 3: Partial Autocorrelation Function (ACF) for Gold Price from January 2015 to December 2017

Based on the partial autocorrelation of the time should be differentiated to function (PACF) in Figure 3, there was a achieve stationary state. truncated spike indicating that the data series

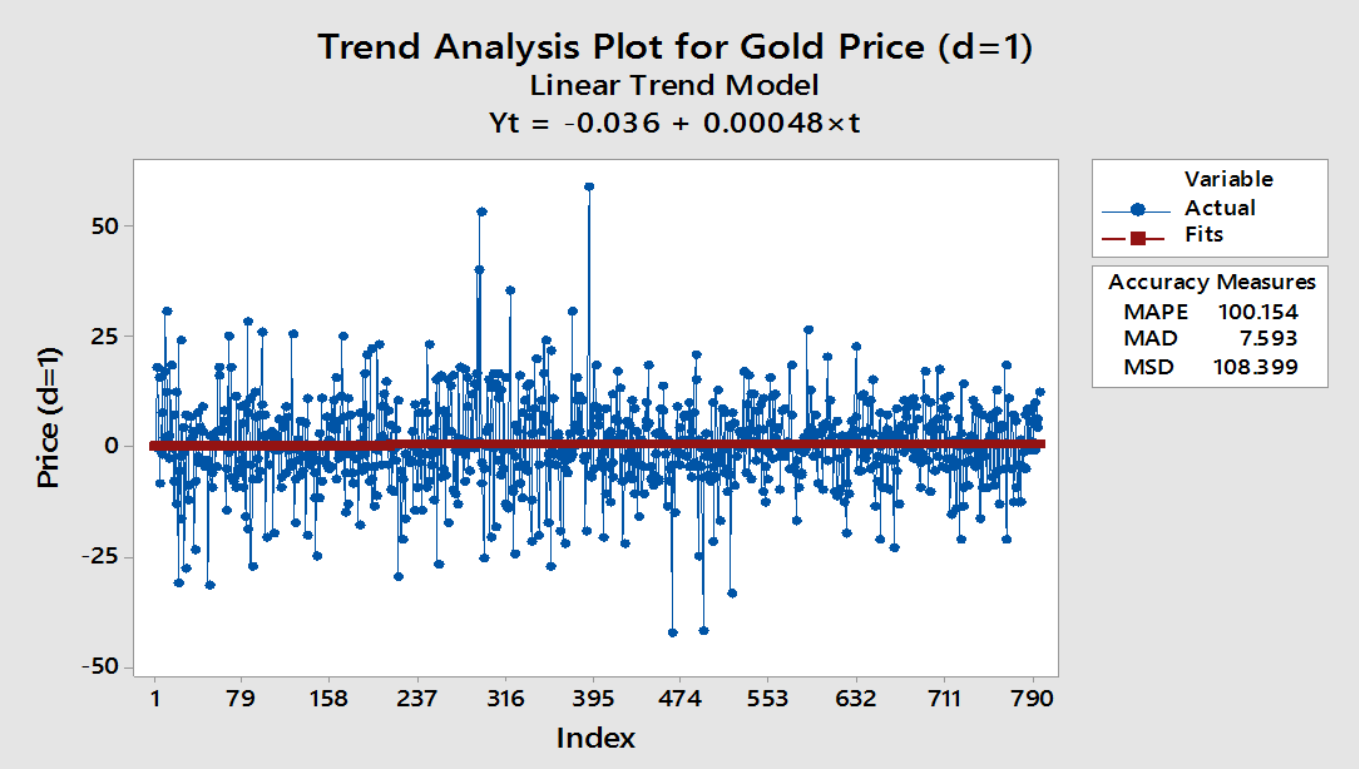

Figure 4: First Differentiation for Time Series of Gold Price (d=1) From January 2015 to December 2017

After first differentiation was done, Figure 4 shows that the red line that means that there was no significant difference between mean value and variance. Therefore, this line shows that the time series were normally distributed with mean value of zero and variance value of one. Thus, this time series was said to be in stationary state. 


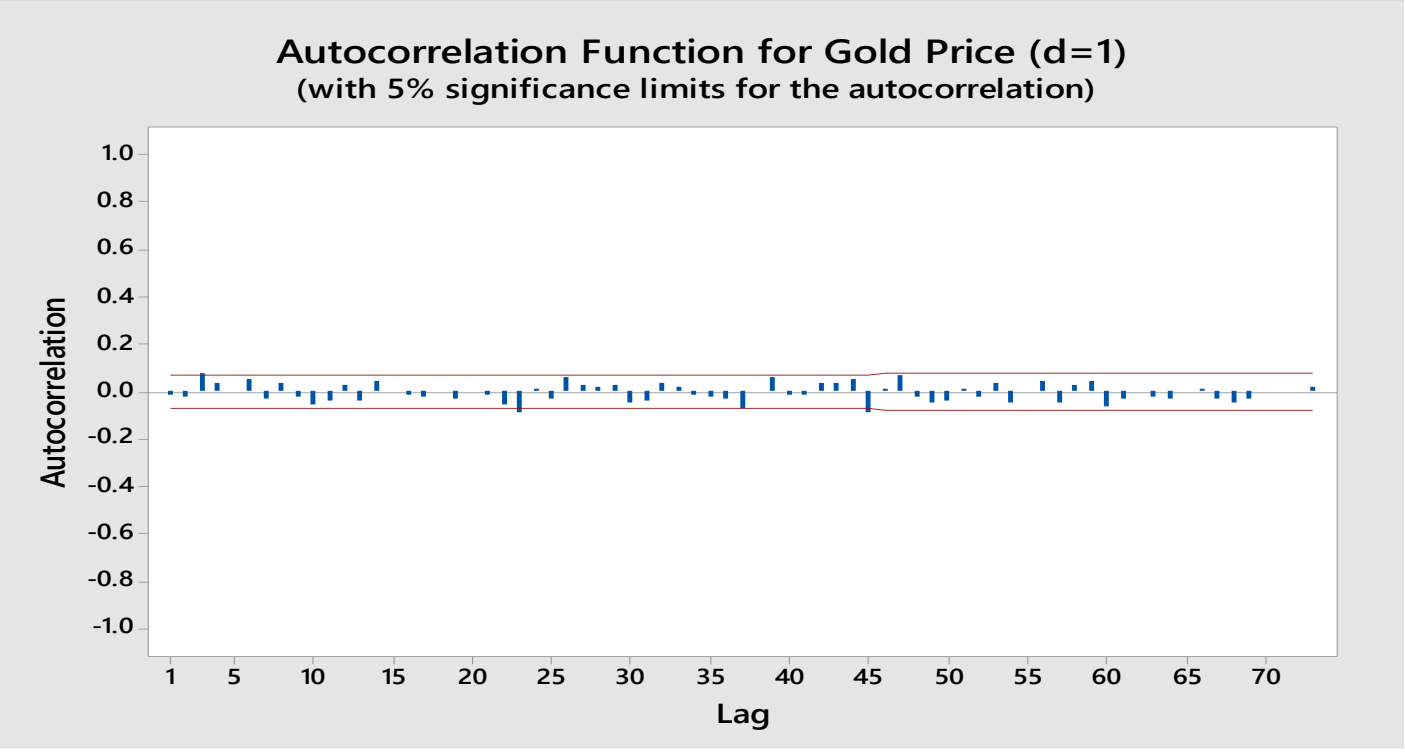

Figure 5: Autocorrelation Function (ACF) for First Differentiation of Gold Price from January 2015 to December 2017

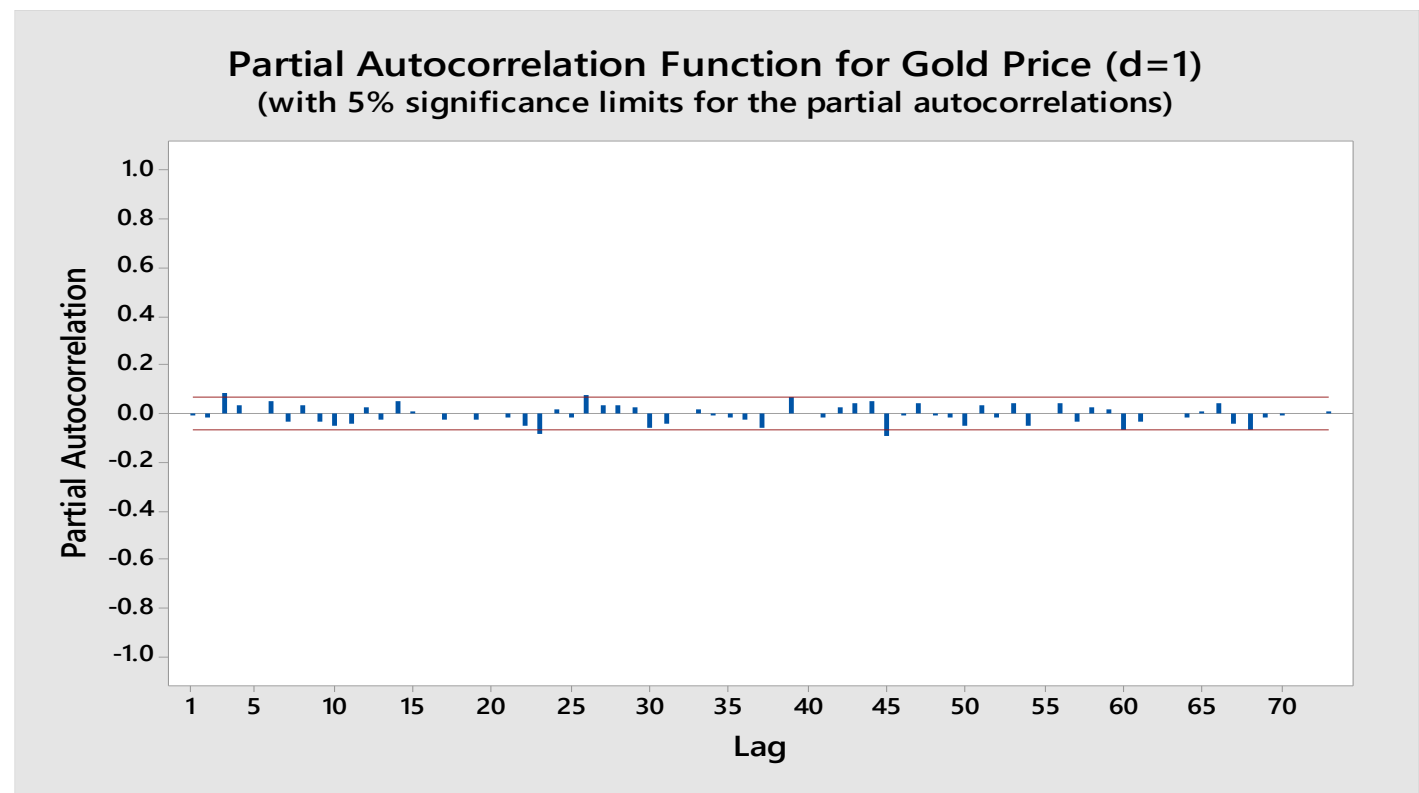

Figure 6: Partial Autocorrelation Function (PACF) for First Differentiation of Gold Price from January 2015 to December 2017

Besides, based on Figure 5 and Figure 6, Autocorrelation Function (ACF) and Partial Autocorrelation Function (PACF) showed drastically decreasing pattern. Thus, it can be concluded that this time series data achieved stationary state after first differentiation was done. By this means, value of $d$ in this ARIMA model $(p, d, q)$ for this time series data was equal to $d=1$.

Determination of model can be known based on correlogram analysis of ACF and
PACF. Based on correlogram analysis of $\mathrm{ACF}$ and PACF, value of parameter $p$ and parameter $q$ can be determined. ARIMA model $(p, d, q)$ can be built by estimating through ARMA model $(p, q)$. After observation was done towards Figure 5 and Figure 6, there are few spikes which exceeded the dotted line for ACF and PACF. AR model and MA model can be determined through number of spikes that exceeded the dotted line which can represent number of $p$ and $q$. Based on Figure 5, it was found that 
the Autocorrelation Function (ACF) had 1 spike while the Partial Autocorrelation Function (PACF) had 1 spike based on Figure 6. Therefore, the model used was ARIMA model $(1,1,1)$.
Next, the ARIMA (1,1,1), ARIMA $(0,1,1)$ and ARIMA $(1,1,0)$ models were obtained through the experimental process using MINITAB software. The correlogram analysis is as follows:

Table 3: Analysis Result of ARIMA $(1,1,1)$

Final Estimates of Parameters

\begin{tabular}{lrrrr} 
Type & Coef & SE Coef & T-Value & P-Value \\
\hline AR 1 & 0.09 & 3.32 & 0.03 & 0.977 \\
MA 1 & 0.10 & 3.31 & 0.03 & 0.975 \\
Constant & 0.140 & 0.332 & 0.42 & 0.674
\end{tabular}

Differencing: 1 regular difference

Number of observations: Original series 795, after differencing 794

Residual Sums of Squares

\begin{tabular}{rrr} 
DF & SS & MS \\
\hline 791 & 86068.5 & 108.810
\end{tabular}

Back forecasts excluded

Modified Box-Pierce (Ljung-Box) Chi-Square Statistic

\begin{tabular}{lrrrr} 
Lag & 12 & 24 & 36 & 48 \\
\hline Chi-Square & 15.16 & 28.22 & 38.82 & 60.31 \\
DF & 9 & 21 & 33 & 45 \\
P-Value & 0.087 & 0.134 & 0.224 & 0.063
\end{tabular}

Table 4: Analysis Result of ARIMA $(0,1,1)$

Final Estimates of Parameters

\begin{tabular}{lrrrr} 
Type & Coef & SE Coef & T-Value & P-Value \\
\hline MA 1 & 0.0099 & 0.0356 & 0.28 & 0.780 \\
Constant & 0.154 & 0.366 & 0.42 & 0.674
\end{tabular}

Differencing: 1 regular difference

Number of observations: Original series 795 , after differencing 794

Residual Sums of Squares

\begin{tabular}{rrr} 
DF & SS & MS \\
\hline 792 & 86070.0 & 108.674
\end{tabular}

Back forecasts excluded

Modified Box-Pierce (Ljung-Box) Chi-Square Statistic

\begin{tabular}{lrrrr} 
Lag & 12 & 24 & 36 & 48 \\
\hline Chi-Square & 15.17 & 28.21 & 38.81 & 60.35 \\
DF & 10 & 22 & 34 & 46 \\
P-Value & 0.126 & 0.169 & 0.262 & 0.076
\end{tabular}


Table 5: Analysis Result of ARIMA $(1,1,0)$

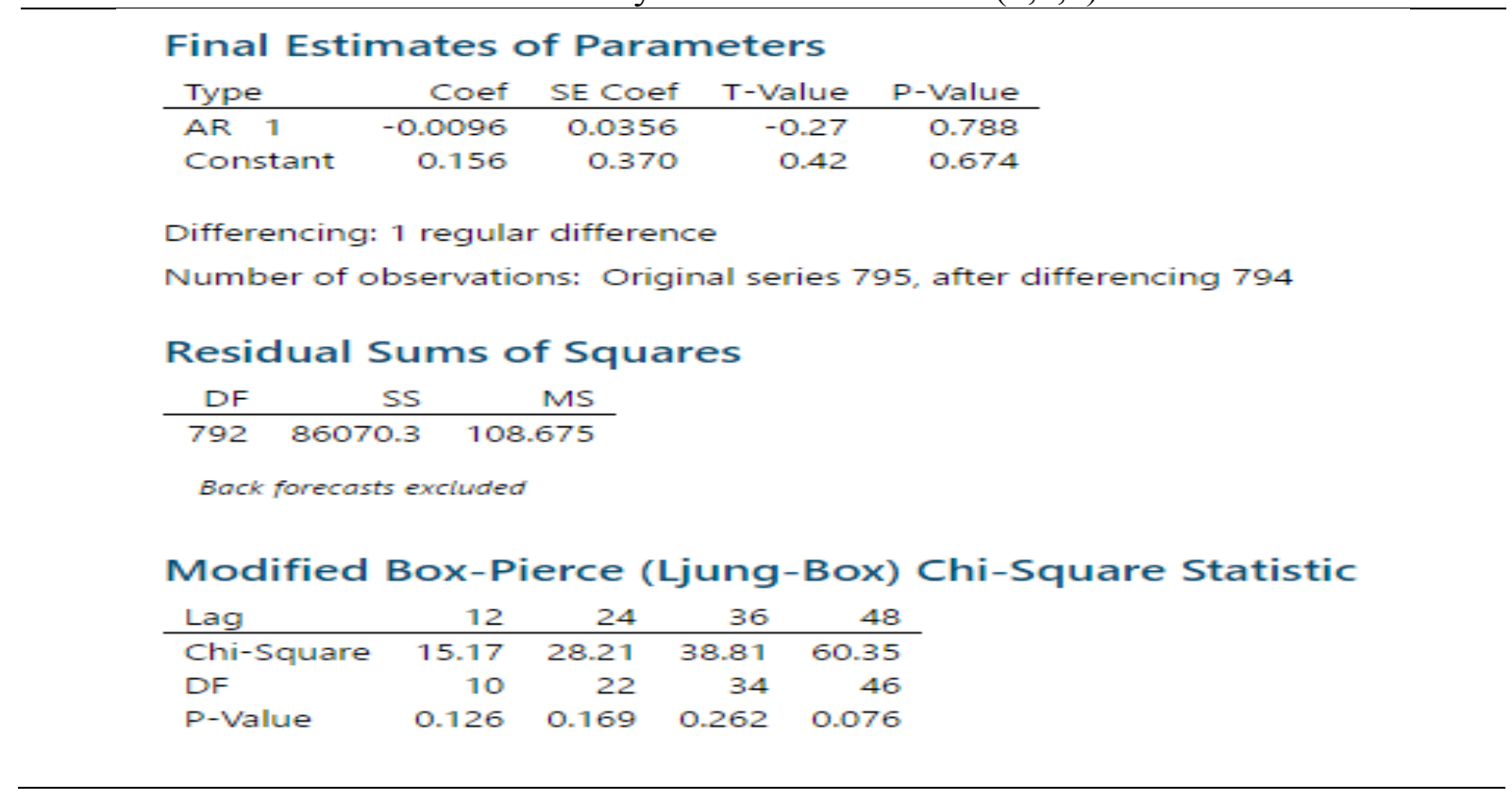

Therefore, ARIMA (1,1,1), ARIMA $(0,1,1)$ and ARIMA $(1,1,0)$ models would continue to the diagnostic checking stage to test the most suitable models for gold commodity price forecasting.

Stage of diagnostic review can be done using Ljung-Box. The checking of the calculated values for the Ljung-Box, $\mathrm{Q} *$ values can be tested by comparing the tabulated values $\mathrm{Q} *$ and calculated $\mathrm{Q} *$ values. As a result of the checking, it was found that all models existed in white noise error. The model to choose is based on the smallest statistic $\mathrm{Q} *$. Table 6 below shows that the ARIMA model $(1,1,1)$ was the most suitable model and better than other models. This is because the ARIMA model $(1,1,1)$ has the smallest MSE value and $\mathrm{Q} *\left(Q^{*}\right.$ calculated $=15.16<Q^{*}$ tabulated $\left.=16.91\right)$. Therefore, due to the ARIMA model $(1,1,1)$ fulfilling the criteria, it can be concluded that the ARIMA model $(1,1,1)$ was chosen as the best model for gold commodity price forecasting.

$H_{0}: \theta=0$ error is random (white noise error)

$H_{1}: \theta \neq 0$ error is not random (no white noise error)

Table 6: Portmanteau Test Summary

\begin{tabular}{cccc}
\hline Statistic & ARIMA $(1,1,1)$ & ARIMA $(0,1,1)$ & ARIMA $(1,1,0)$ \\
\hline $\begin{array}{c}\text { Calculated } Q^{*} \\
\text { Ljung-Box Value) }\end{array}$ & 15.16 & 15.17 & 15.17 \\
\hline DF & 9 & 10 & 18.30 \\
\hline $\begin{array}{c}\text { Tabulated } Q^{*} \\
\text { Ljung-Box Value })\end{array}$ & 16.91 & 18.30 & Accept $H_{0}$ \\
\hline $\begin{array}{c}\text { Decision (5\% } \\
\text { significance limits) }\end{array}$ & Accept $H_{0}$ & Accept $H_{0}$ & Error is a white noise \\
\hline Conclusion & Error is a white noise & Error is a white noise & 116.250 \\
\hline MSE & 114.058 & 116.069 & \\
\hline
\end{tabular}


Forecasting for gold price was continued using ARIMA model $(1,1,1)$ since it was the best model.

$\phi(B)(1-B)^{d} X_{t}=\theta(B) Z_{t}$

or

$\left(1-\emptyset_{1} B-\emptyset_{2} B^{2}-\cdots-\emptyset_{P} B^{P}\right)(1-B)^{d} X_{t}=\mu+\left(1-\theta_{1} B-\theta_{2} B^{2}-\cdots-\theta_{P} B^{P}\right) Z_{t}$

Generally, ARIMA model $(p, d, q)$ can be summarized as:

where $X_{t}$ represents the value of the time series based on time and $Z_{t}$ represents stationary time series.

Thus, the equation for ARIMA model $(1,1,1)$ is as follows:

$$
(1-0.09 B)(1-B) X_{t}=0.140+(1-0.10 B) Z_{t}
$$

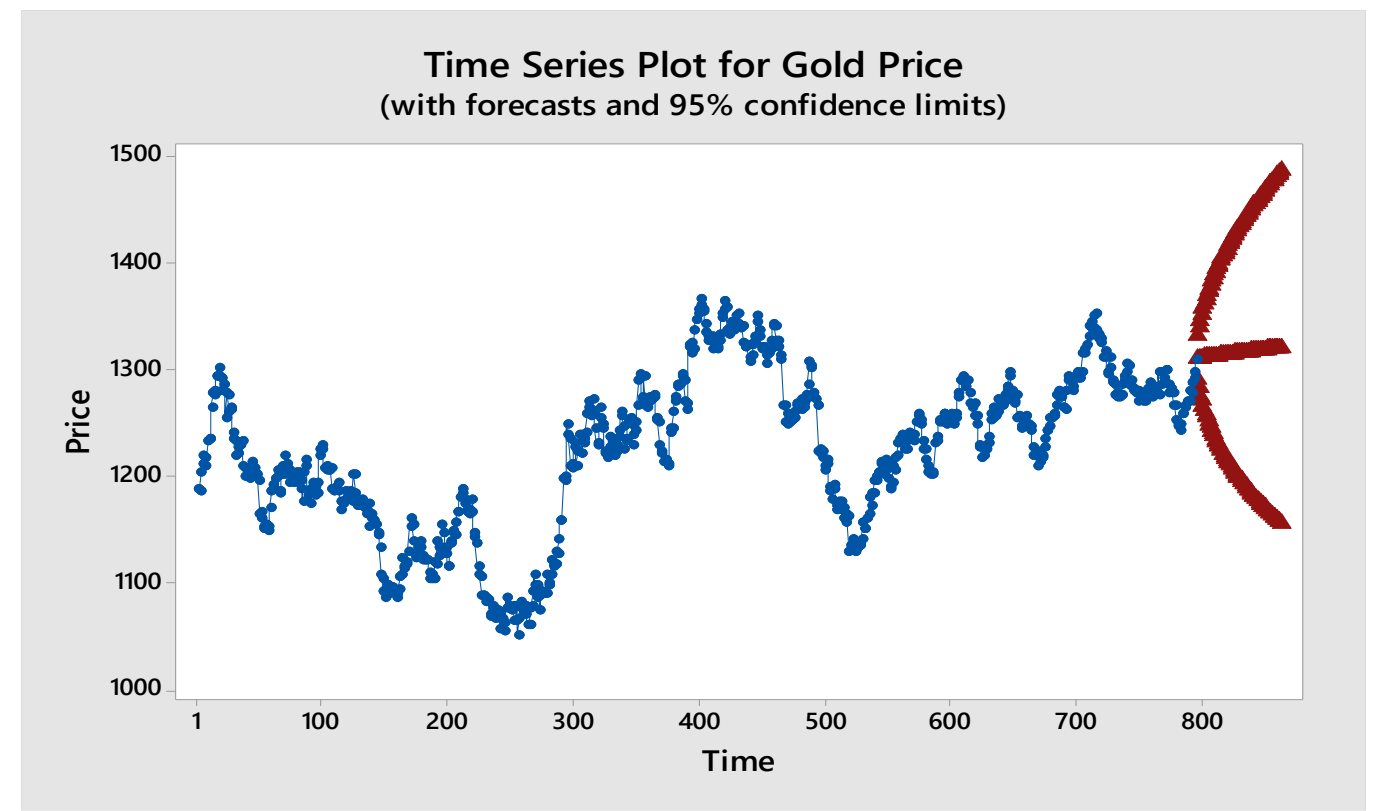

Figure 7: Gold Price Forecasting in January 2018 to March 2018

Based on Figure 7, gold prices for future are expected to increase. 


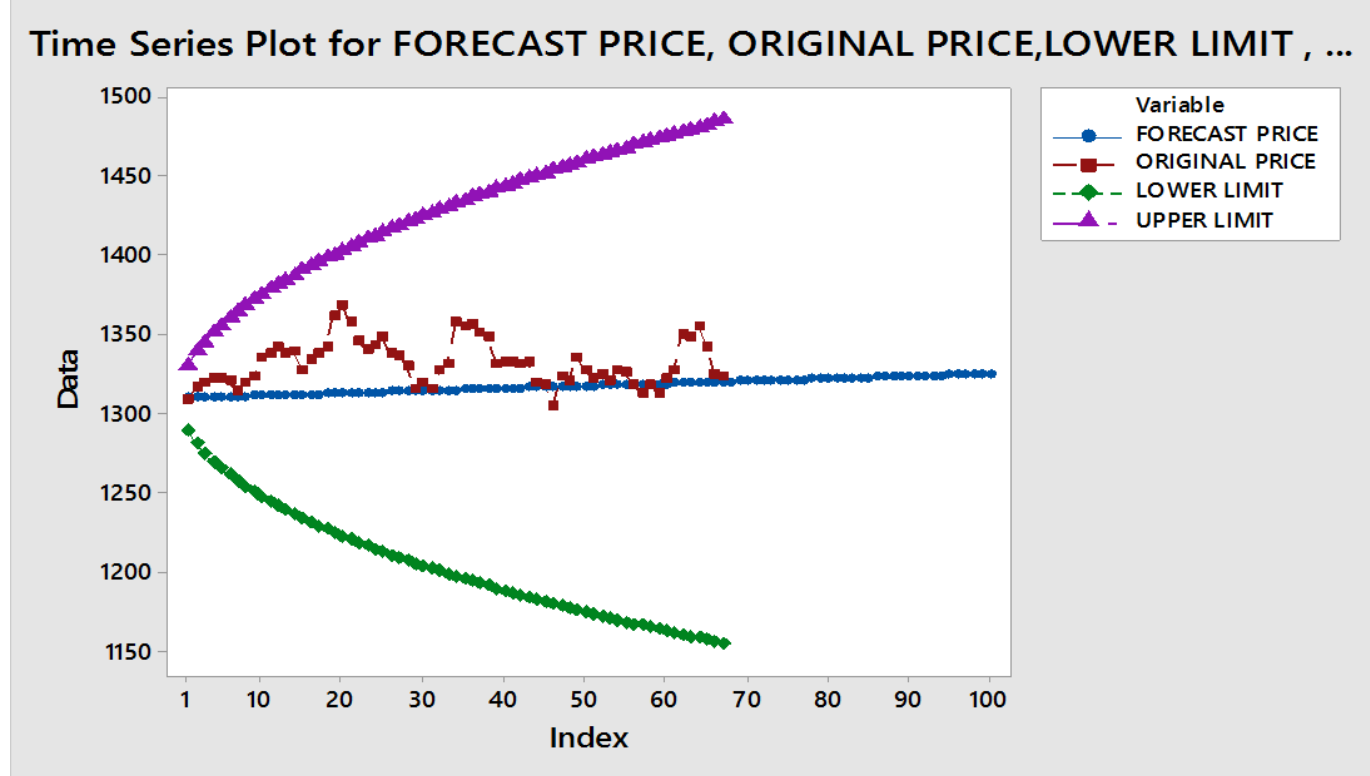

Figure 8: Comparison of Gold Price between Original Value and Forecast Value

Based on Figure 8, comparison of gold price between original value and forecast value was obtained.

MAPE formula is given as follows:

$$
\begin{aligned}
\text { MAPE } & =\frac{\left|\left(\frac{1145.03}{89209.3}\right) * 100\right|}{67} \\
& =0.02 \%
\end{aligned}
$$

Based on the MAPE value that has obtained which is $0.02 \%$, it is found that ARIMA $(1,1,1)$ is the best model for predicting gold commodity prices.

Based on ARIMA model $(1,1,1)$, forecast result of gold prices for 2018 are expected to increase. Thus, world gold commodity performance in 2018 can be analyzed using the best ARIMA model $(1,1,1)$.

\section{Conclusion}

The objective of this research is to forecast world gold commodity prices in 2018 by using the Box-Jenkins method. Using BoxJenkins method, the process of forecasting can be done to identify the movement of gold commodity prices whether to achieve a rise or fall in the future. Therefore, this forecasting model can help the various groups involved, especially the investors. This forecast will also help companies involved in selling and buying gold.
Additionally, this forecast will help companies involved in gold investment in the development of gold buying and selling system.

In conclusion, based on the analysis conducted, ARIMA $(1,1,1)$ model was the best model in forecasting world gold commodity prices in 2018. Therefore, using the ARIMA model $(1,1,1)$ gold commodity price forecasting for January 2018 to March 2018 was made. Through the forecasting, it was found that gold commodity prices would be rising in the first 3 months in 2018 as shown in Figure 7 in the Discussion section.

\section{Acknowledgements}

The authors would like to express gratitude to the School of Informatics and Applied Mathematics (PPIMG) and Universiti Malaysia Terengganu (UMT) for the support given. 


\section{References}

Ali, A., Muhammad Iqbal, C., Sadia, Q., Noureen, A.,Tahir, M., Hyder, M., dan Muhammad Tariq, J. (2016). Forecasting Of Daily Gold Price By Using Box-Jenkins Methodology, International Journal of Asian Social Science, 6(11): 614-624.

Lewis (1982). MAPE CRITERIA FOR MODEL EVALUATION, Forecasting Power. Researchgate.net., 25(4): 500-506

Mohamad Alias, L. (2011). Introductory Business Forecasting: A Practical
Approach. Ed. Ke-3. Shah Alam: Penerbit Universiti Teknologi MARA.

NIAGA HADAPAN EMAS (2017 September 13).Data Emas Dunia. Nukilan daripada https://www.investing.com/commodities gold-historical-data

Kusumadewi, F. (2014). Peramalan Harga Emas Menggunakan Feedforward Neural Network Dengan Algoritma Backpropagation, Fakultas Matematika dan Ilmu Pengetahuan Alam Universitas Negeri Yogyakarta. 